\title{
Photoinduced melting of magnetic order in the correlated electron insulator $\mathrm{NdNiO}_{3}$
}

\author{
A. D. Caviglia, ${ }^{1,2}$ M. Först, ${ }^{1}$ R. Scherwitzl,${ }^{3}$ V. Khanna,,${ }^{1,4,5}$ H. Bromberger, ${ }^{1}$ R. Mankowsky, ${ }^{1}$ R. Singla, ${ }^{1}$ Y.-D. Chuang, ${ }^{6}$
} W. S. Lee, ${ }^{7}$ O. Krupin, ${ }^{8}$ W. F. Schlotter, ${ }^{9}$ J. J. Turner, ${ }^{9}$ G. L. Dakovski, ${ }^{9}$ M. P. Minitti, ${ }^{9}$ J. Robinson, ${ }^{9}$ V. Scagnoli, ${ }^{10}$ S. B. Wilkins, ${ }^{11}$ S. A. Cavill, ${ }^{5,}$ M. Gibert,${ }^{3}$ S. Gariglio, ${ }^{3}$ P. Zubko, ${ }^{3}$ J.-M. Triscone, ${ }^{3}$ J. P. Hill, ${ }^{11}$ S. S. Dhesi,${ }^{5}$ and A. Cavalleri ${ }^{1,4}$

${ }^{1}$ Max-Planck Institute for the Structure and Dynamics of Matter, Hamburg, Germany

${ }^{2}$ Kavli Institute of Nanoscience, Delft University of Technology, Delft, The Netherlands

${ }^{3}$ Département de Physique de la Matière Condensée, University of Geneva, Geneva, Switzerland

${ }^{4}$ Department of Physics, Clarendon Laboratory, University of Oxford, Oxford, United Kingdom

${ }^{5}$ Diamond Light Source, Chilton, Didcot, Oxfordshire, United Kingdom

${ }^{6}$ Advanced Light Source, Lawrence Berkeley Laboratory, Berkeley, California 94720, USA

${ }^{7}$ The Stanford Institute for Materials and Energy Sciences (SIMES), Stanford Linear Accelerator Center (SLAC) National Accelerator Laboratory, and Stanford University, Menlo Park, California 94305, USA

${ }^{8}$ European XFEL GmbH, Hamburg, Germany

${ }^{9}$ Linac Coherent Light Source, Stanford Linear Accelerator Center (SLAC) National Accelerator Laboratory, Menlo Park, California 94305, USA

${ }^{10}$ Swiss Light Source, Paul Scherrer Institute, CH-5232 Villigen PSI, Switzerland

${ }^{11}$ Condensed Matter Physics and Materials Science Department, Brookhaven National Laboratory, Upton, New York 11973, USA

(Received 8 April 2013; published 2 December 2013)

\begin{abstract}
Using ultrafast resonant soft $\mathrm{x}$-ray diffraction, we demonstrate photoinduced melting of antiferromagnetic order in the correlated electron insulator $\mathrm{NdNiO}_{3}$. Time-dependent analysis of the resonant diffraction spectra allows us to follow the temporal evolution of the charge imbalance between adjacent $\mathrm{Ni}$ sites. A direct correlation between the melting of magnetic order and charge rebalancing is found. Furthermore, we demonstrate that the magnetic ordering on the $\mathrm{Ni}$ and $\mathrm{Nd}$ sites, which are locked together in equilibrium, become decoupled during this nonthermal process.
\end{abstract}

DOI: 10.1103/PhysRevB.88.220401

PACS number(s): 78.20.Ls, 73.90.+f, 78.47.-p, 78.70.Ck

Ultrafast photomagnetism, the use of light to control the spin arrangements of solids, may open the way to high-speed switching in new data storage devices. For example, femtosecond manipulation of magnetic order has been demonstrated in various metallic ferromagnetic systems, ${ }^{1-4}$ in which the spin dynamics result from thermal coupling to photoexcited hot electrons (for a review, see Ref. 5).

Further investigations have focused on the photomagnetism of strongly correlated electron systems. Work has been done on antiferromagnetic compounds, such as $\mathrm{NiO}$ and $\mathrm{Fe}$ pnictides, using time-resolved second-harmonic generation or photoemission. ${ }^{6,7}$ In most complex oxides, photoinduced demagnetization is understood as a direct consequence of charge redistributions between different orbitals. Here, prompt perturbation in the orbital occupancy is expected to affect the pattern of exchange interactions ${ }^{8}$ in the spirit of GoodenoughKanamori-Anderson rules. ${ }^{9}$ Recent time-resolved experiments have combined ultrafast optical excitation with resonant soft x-ray diffraction ${ }^{10-14}$ and have received particular impetus by the availability of $\mathrm{x}$-ray free-electron lasers (XFELs). However, to date, direct experimental proof of the correlation between magnetic and charge/orbital dynamics still needs to be obtained.

In this Rapid Communication, we perform ultrafast resonant soft $\mathrm{x}$-ray magnetic scattering during the light-induced melting of antiferromagnetic order in the undoped perovskite neodymium nickelate $\left(\mathrm{NdNiO}_{3}\right)$. By analyzing the incident energy dependence of the scattering, we monitor the evolution of the charge imbalance between two sets of inequivalent Ni sites. This charge disproportionation underpins the antiferromagnetic order at equilibrium. We find a direct correlation between a transient charge rebalance and the melting of the magnetic order. Also, on ultrafast time scales, the $\mathrm{Ni}$ and $\mathrm{Nd}$ sublattices unlock from each other, in contrast to the equilibrium behavior.

In equilibrium, $\mathrm{NdNiO}_{3}$ exhibits a metal-to-insulator transition concomitantly with the appearance of antiferromagnetic order at the Néel temperature $T_{\mathrm{N}}=T_{\mathrm{MI}}{ }^{15,16}$ In thin films this temperature is determined by the epitaxial strain imposed by the substrate. ${ }^{17}$ The microscopic physics of the lowtemperature insulating antiferromagnetic ground state $(T<$ $T_{\mathrm{MI}}$ ) can be summarized as follows. A small gap (about $0.2 \mathrm{eV}$ ) is formed between two bands that originate from strongly hybridized $\mathrm{Ni} 3 d e_{g}$ and $\mathrm{O} 2 p$ orbitals. ${ }^{18-20} \mathrm{~A}$ structural distortion is associated with charge localization onto two sets of $\mathrm{Ni}$ sites, $\mathrm{Ni}_{1}$ and $\mathrm{Ni}_{2} \cdot{ }^{21-24}$ These sites become inequivalent because of the monoclinic symmetry of the low-temperature phase. ${ }^{25}$ As shown in Fig. 1(a), Ni ${ }_{1}$ $\left(\mathrm{Ni}_{2}\right)$ atoms are then surrounded by a large (small) oxygen octahedron, with the on-site charge density being determined by a $4 \%$ difference between the average $\mathrm{Ni}-\mathrm{O}$ bond distances (charge disproportionation). Concomitantly, the oxygen anions become electron deficient, and host localized holes even in the absence of chemical doping. ${ }^{26}$ The resulting exchange pattern produces antiferromagnetic $\operatorname{order}^{26}$ at the $(1 / 41 / 4$ $1 / 4$ ) wave vector in the pseudocubic notation, as visualized in real space in Fig. 1(b) ${ }^{27}$ Furthermore, the magnetic moments in the unfilled $f$ shell of the $\mathrm{Nd}$ atoms order at the same wave vector, driven by the mean field of the neighboring $\mathrm{Ni}$ ions. $^{27,28}$ 
(a)
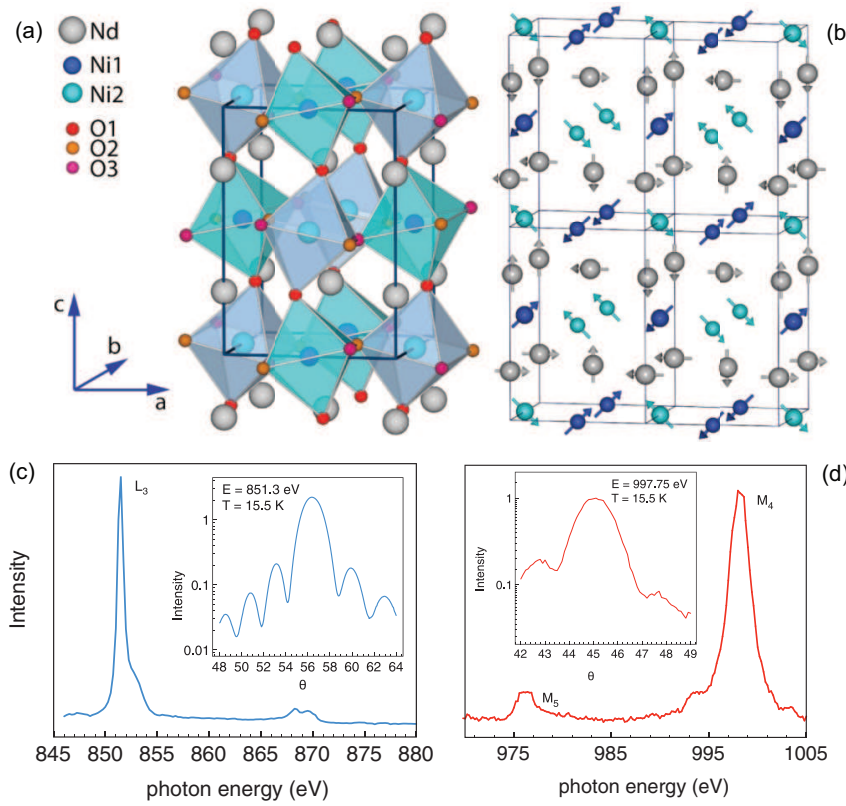

FIG. 1. (Color online) Multiple orders in the low-temperature insulating phase of $\mathrm{NdNiO}_{3}$. (a) Monoclinic crystal structure as measured by high-resolution x-ray diffraction (Ref. 21). In this configuration $\mathrm{Ni}_{1}\left(\mathrm{Ni}_{2}\right)$ atoms are surrounded by a large (small) oxygen octahedron. (b) Magnetic structure of $\mathrm{NdNiO}_{3}$ consistent with resonant soft $\mathrm{x}$-ray diffraction data. Both $\mathrm{Nd}$ and $\mathrm{Ni}$ magnetic moments are ordered. (c) Energy dependence of the magnetic reflection at the $\mathrm{Ni} L_{2,3}$ absorption edge measured at $T=15 \mathrm{~K}$. Inset: Angular dependence ( $\theta-2 \theta$ scan) of the magnetic reflection measured at the maximum intensity of the Ni $L_{3}$ edge. (d) Energy dependence of the magnetic reflection at the $\mathrm{Nd} M_{4,5}$ absorption edge measured at $T=15 \mathrm{~K}$. Inset: Angular dependence $(\theta-2 \theta$ scan $)$ of the magnetic reflection measured at the maximum intensity of the $\mathrm{Nd} M_{4}$ edge.

For the experiment reported here, $\mathrm{NdNiO}_{3}$ thin films, 100 unit cells thick, were deposited on (111) $\mathrm{LaAlO}_{3}$ substrates by off-axis $\mathrm{rf}$ magnetron sputtering. ${ }^{29}$ The epitaxial strain imposed by the substrate determines $T_{\mathrm{MI}}=150 \mathrm{~K}$. Below this temperature antiferromagnetic order sets in, as is directly evidenced by resonant soft $\mathrm{x}$-ray diffraction measurements. ${ }^{30}$ We carried out optical pump, resonant soft $\mathrm{x}$-ray diffraction probe experiments at two sources. The first, the I06 end station of the Diamond Light Source (DLS), combines 70 ps time resolution (limited by the duration of the x-ray probe pulses) with very high energy resolution, $10^{4}$ resolving power, to measure the evolution of the resonant diffraction line shape. The second, at the SXR beamline ${ }^{31,32}$ of the Linac Coherent Light Source (LCLS) free-electron laser, provides an extremely good time resolution of $200 \mathrm{fs}$ as determined by the timing jitter between the $\mathrm{x}$-ray and the optical laser pulses. In each case, the sample was excited by $800 \mathrm{~nm}, 100 \mathrm{fs}$ pulses impinging collinearly and temporally synchronized with the $\mathrm{x}$-ray pulses, and the scattering at the $(1 / 41 / 41 / 4)$ wave vector was detected with an avalanche photodiode at each pump-probe time delay. The pump photon energy $(1.5 \mathrm{eV})$ matches a broad infrared band assigned to transitions from $t_{2 g}$-like to $e_{g}$-like states. ${ }^{33}$ Although on-site transitions of this type are not dipole allowed in a $d$ shell, which is only split by the electrostatic forces of the crystal field, hybridization with the neighboring oxygen orbitals makes this direct excitation possible in the present case.

Figure 1(c) shows the energy dependence of the $(1 / 4$ $1 / 41 / 4)$ magnetic reflection at the $\mathrm{Ni} L_{2,3}$ absorption edges measured at $T=15 \mathrm{~K}$. The inset of Fig. 1(c) shows the angular dependence $(\theta-2 \theta$ scan) of the magnetic reflection, measured at the maximum intensity of the $\mathrm{Ni} L_{3}$ edge $(851.3 \mathrm{eV})$. The observation of interference fringes along the crystal truncation rod is indicative of high structural and magnetic coherence. Finally, the angular and energy dependence of the magnetic reflection measured at the $\mathrm{Nd} M_{4,5}$ edges is shown in Fig. 1(d). The origin of these peaks is also purely magnetic and reflects the ordering of the Nd magnetic moments. ${ }^{27}$

We first discuss the temporal evolution of the resonant line shape at the $\mathrm{Ni} L_{3}$ edge that corresponds to a $2 p-3 d$ electric dipole transition. The $\mathrm{x}$-ray photon energy dependence of this scattering signal contains information on the electronic states of the $3 d$ shell. Charge transfer multiplet calculations show that these spectra are best described by considering the charge disproportionated state as a superposition of electron configurations $\alpha\left(3 d^{7} 2 p^{6}\right)+\beta\left(3 d^{8} 2 p^{5}\right)$, with $\alpha>\beta$ for $\mathrm{Ni}_{1}$ and $\alpha<\beta$ for $\mathrm{Ni}_{2} .{ }^{30}$ The $\left(3 d^{8} 2 p^{5}\right)$ state corresponds to two $e_{g}$ electrons on the $\mathrm{Ni}$ site and one hole in the oxygen ligands. The presence of ordered and localized holes in the absence of chemical doping is a direct consequence of the strong hybridization of $\mathrm{Ni} 3 d$ and $\mathrm{O} 2 p$ levels. ${ }^{20}$

Figure 2(a) shows the time- and energy-resolved intensity of the (1/4 1/4 1/4) magnetic reflection at the $\mathrm{Ni} L_{3}$ edge after
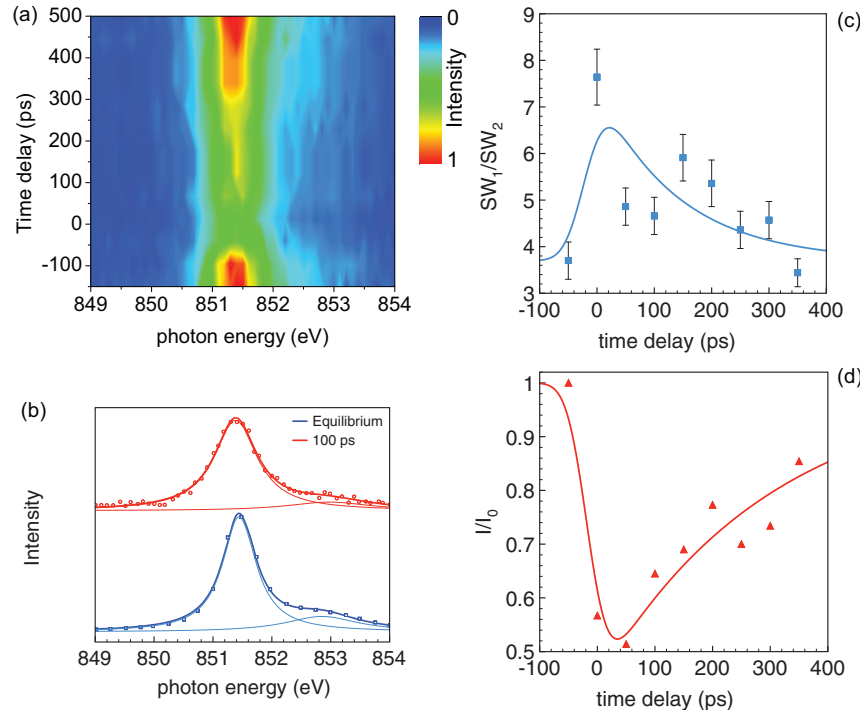

FIG. 2. (Color online) Ultrafast charge rebalancing in $\mathrm{NdNiO}_{3}$. (a) Evolution of the energy-resolved intensity of the $(1 / 41 / 41 / 4)$ magnetic reflection around the $\mathrm{Ni} L_{3}$ edge after ultrafast laser excitation (100 fs, $\left.800 \mathrm{~nm}, 1.5 \mathrm{~mJ} / \mathrm{cm}^{2}\right)$. (b) Example of best fits of two Lorentzian peaks to individual spectra measured at different time delays, which allows the determination of the energy-integrated scattering intensity of the individual peaks $\left(\mathrm{SW}_{1}, \mathrm{SW}_{2}\right)$. (c) Scatter points: Evolution of the ratio of the energy-integrated scattering intensity of the two Lorentzian peaks after photoexcitation. Solid line: Guide to the eye. (d) Scatter points: Evolution of the normalized peak intensity $I / I_{0}$ after photoexcitation. Solid line: Exponential fit to the data. 
ultrafast laser excitation (fluence $F=1.5 \mathrm{~mJ} / \mathrm{cm}^{2}$ ). Figure 2(b) provides a focused view on the $\mathrm{Ni} L_{3}$ resonance in thermal equilibrium and at a positive time delay. A satellite is seen on the main peak and individual spectra are well described by two Lorentzian envelopes of different heights and widths. The splitting of the satellite at the $\mathrm{Ni} L_{3}$ peak quantifies the degree of charge disproportionation $\delta=\alpha_{\mathrm{Ni}_{1}}-\alpha_{\mathrm{Ni}_{2}}$. ${ }^{34}$ Here we characterize these two peaks by their energy-integrated intensities $\mathrm{SW}_{1}$ and $\mathrm{SW}_{2}$.

The central message of this Rapid Communication is that the photoinduced dynamics of the antiferromagnetic order parameter involve a redistribution of spectral weight across these two peaks. Figure 2(c) shows the evolution of the ratio of the energy-integrated intensities of the two peaks after optical excitation. A reshaping of the spectral features is apparent, with a transient increase in the peak asymmetry as quantified by $\mathrm{SW}_{1} / \mathrm{SW}_{2}$. The relaxation of the spectral features back to equilibrium takes place on a similar time scale as the peak intensity, as shown in Fig. 2(d). It is important to note that the time resolution of this experiment is limited by the duration of the X-ray probe pulses at DLS (70 ps) and the intrinsic time scale of the underlying transient dynamics cannot be accessed.

The microscopic physics of such charge melting can be understood as follows. As discussed above, photoexcitation occurs within each site from $t_{2 g}$-like to $e_{g}$-like states. ${ }^{35}$ This charge transfer is expected to lead to a significant perturbation of the local wave function and a concomitant change in the hybridization with the surrounding oxygen ligands that will alter the equilibrium disproportionation of charge. According to Mizokawa et al. ${ }^{26}$ this charge ordering is strongly coupled to the antiferromagnetic order on the $\mathrm{Ni}$ sites. Thus, the observed melting of the magnetic order must be the direct consequence of the transient photoinduced change of the charge disproportionation.

The interplay between charge transfer and magnetization dynamics has not been accessible in earlier work on, e.g., photoinduced charge/orbital and magnetic order melting in manganites. ${ }^{10}$ Note further that the driving mechanism for this charge redistribution and the resulting magnetic order melting is a nonthermal process (see also Refs. 10 and 36). Whether the transient state remains insulating or becomes metallic is an open question to be addressed experimentally, possibly through time-resolved $\mathrm{THz}$ optical spectroscopy. Another possibility is that a change in lattice structure also triggers a rearrangement in the low-temperature atomic distortion along a coherent lattice coordinate, a phenomenon often seen in correlated electron systems. ${ }^{37}$ Ultrahigh temporal resolution ( 10fs) hard x-ray diffraction experiments would be needed to clarify this issue, a feat still beyond the technical capabilities of current XFEL technology.

With the understanding that the microscopic photomelting process involves a charge redistribution, we now turn to detailed measurements of the ultrafast melting, as observed with femtosecond x-ray diffraction at the $\mathrm{Ni} L_{3}$ and $\mathrm{Nd}$ $M_{4}$ edges $(T=40 \mathrm{~K})$. These two edges allow the ultrafast dynamics of the two magnetic sublattices to be probed independently.

In the fast dynamics, two distinct response regimes were found, dependent on the excitation fluence. We first discuss the low-fluence regime. Figure 3(a) shows the evolution of the
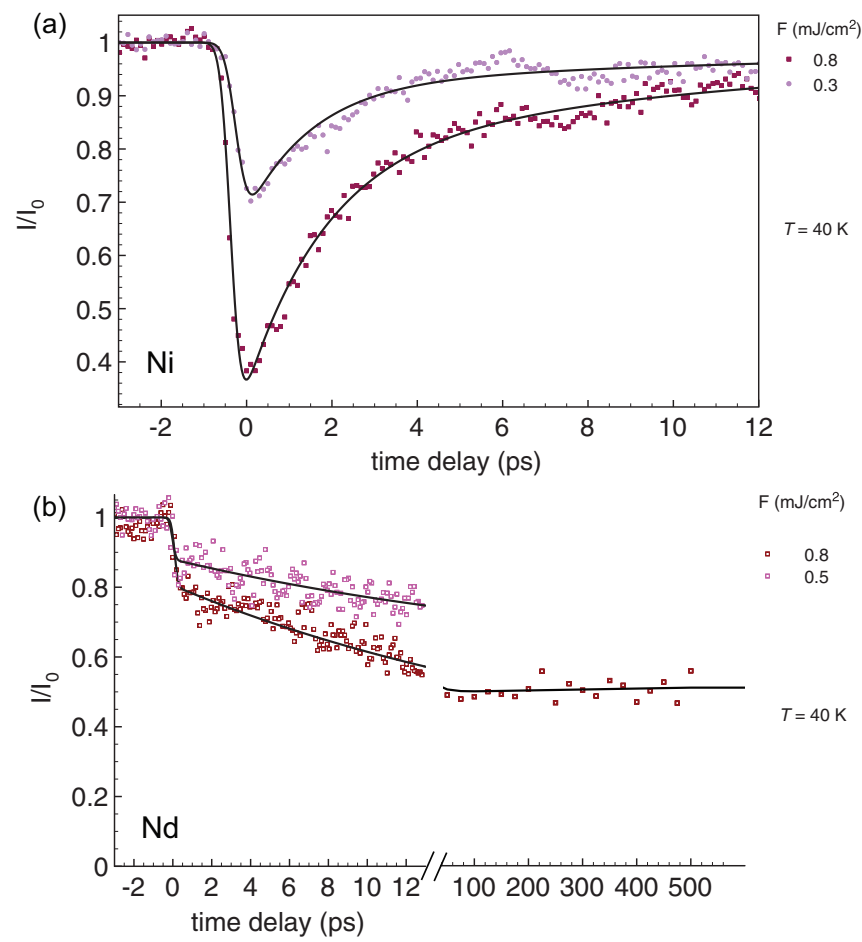

FIG. 3. (Color online) Photomagnetism in $\mathrm{NdNiO}_{3}$ for low excitation fluence. Scatter points: Evolution of the intensity $I$ of the (a) Ni and (b) Nd magnetic diffraction peak, with respect to the equilibrium intensity $I_{0}$, observed for different near-infrared laser fluences. Solid lines: Best fits to double exponential functions.

normalized intensity $I / I_{0}$ of the magnetic $\mathrm{Ni} L_{3}$ diffraction peak for $F<1 \mathrm{~mJ} / \mathrm{cm}^{2}$. A prompt reduction in diffraction intensity is observed, followed by a double exponential recovery with a slow $\tau_{1}$ in the 10 ps range and a faster $\tau_{2} \sim 2$ ps. Figure 3(b) displays the dynamics measured at the same wave vector for probe wavelengths tuned to the $M_{4}$ edge of $\mathrm{Nd}$. The magnetic order on the $\mathrm{Nd}$ sites is also promptly reduced after the optical excitation, but it drops further on the picosecond time scale and remains melted well beyond the 500 ps range of the experiment. Thus, unlike what is observed at thermal equilibrium, the magnetic orders on the $\mathrm{Nd}$ and $\mathrm{Ni}$ sites become unlocked on the ultrafast time scale. We will return to this point below. We note that such differences in the ultrafast dynamics of magnetic sublattices have been reported earlier in photoexcited ferrimagnets, although in these cases the demagnetization is driven by an increase of the electronic temperature, ${ }^{38}$ and not by a charge transfer.

At higher fluences $\left(F>1 \mathrm{~mJ} / \mathrm{cm}^{2}\right)$ complete melting of the magnetism is found. Figures 4(a) and 4(b) show the evolution of the two sublattices in this regime. An almost complete suppression of magnetic signal is observed for both $\mathrm{Ni}$ and $\mathrm{Nd}$ sublattices. As was observed for the lower fluences, the drop in diffraction intensity is prompt for the $\mathrm{Ni}$ sites but the $\mathrm{Nd}$ sublattice has a second slower time constant, which decreases with increasing fluence. The high-fluence recovery dynamics in $\mathrm{Ni}$ is a single exponential with a slow time constant $\tau_{1}>100 \mathrm{ps}$ [Fig. 4(a)]. A summary of the Ni recovery times for different excitation fluences is shown in the inset of Fig. 4(a). The high-fluence recovery of the $\mathrm{Nd}$ magnetic order, as shown in 

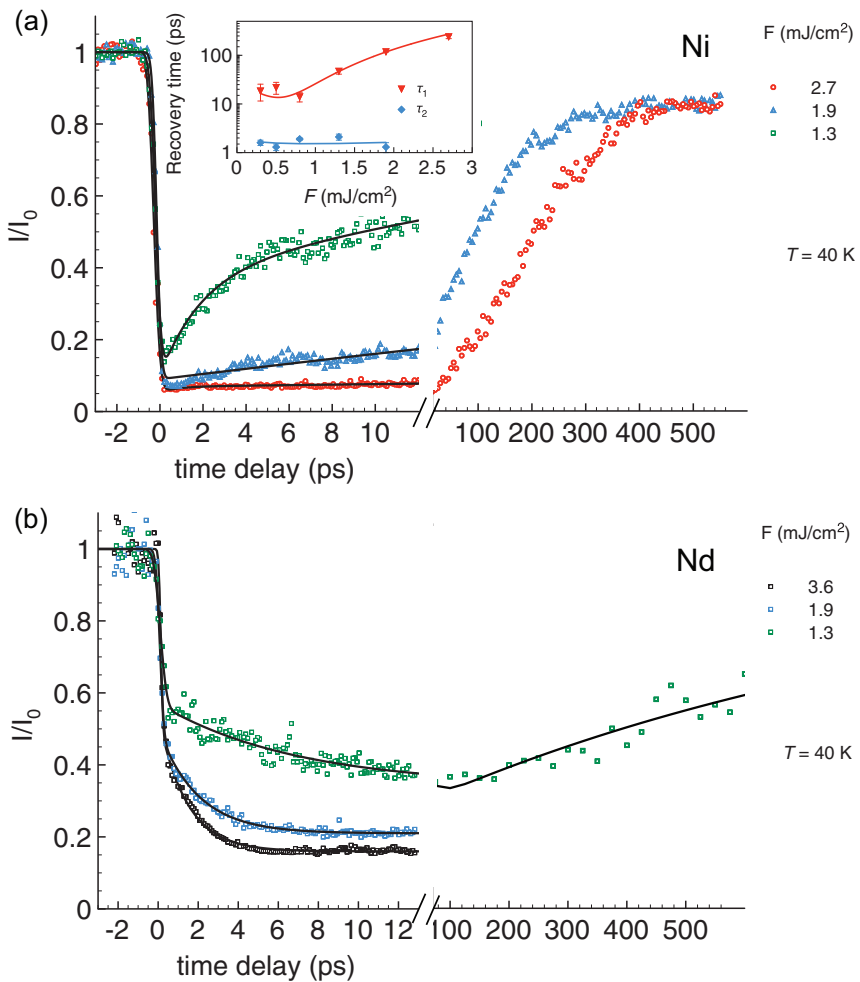

FIG. 4. (Color online) Photomagnetism in $\mathrm{NdNiO}_{3}$ for high excitation fluence. Scatter points: Evolution of the intensity $I$ of the (a) $\mathrm{Ni}$ and (b) Nd magnetic diffraction peak, with respect to the equilibrium intensity $I_{0}$, observed for different near-infrared laser fluences. Solid lines: Best fits to exponential functions. (a) Inset, scatter points: Slow and fast exponential recovery times of $\mathrm{Ni}\left(\tau_{1}, \tau_{2}\right)$ as a function of excitation.

Fig. 4(b), occurs on a slower but comparable time scale to that of $\mathrm{Ni}$. This is in contrast to the low-fluence range, where the dynamics were completely unlocked.

As discussed above, the Nd sublattice appears to respond to an impulsive stimulus and then decays on a slower time scale. This may be due to a direct excitation of the $\mathrm{Nd}^{3+}$ ions occurring within the $4 f$ shell, from the ground state ${ }^{4} I_{9 / 2}$ to a ${ }^{4} F_{5 / 2}$ excited state. However, this is an electric quadrupole transition allowed by spin-orbit coupling and, although it would bring a change in the magnitude of the local magnetic moment, its cross section is likely too weak to explain the size of the observed signal. Rather, the magnetic response of these energetically isolated $\mathrm{Nd} f$ shells can be probably assigned to a prompt reduction in the uncompensated magnetic field that stabilizes order on the $\mathrm{Nd}$ sites at equilibrium. This is expected to exert a torque on the spin alignment, triggering dynamics at a rate that depends on the strength of the magnetic impulse, thus becoming faster as the laser fluence is increased [Fig. 4(b)].

In summary, by combining femtosecond resonant soft x-ray diffraction with high-resolution spectroscopic analysis, we have demonstrated that magnetic melting in the undoped compound $\mathrm{NdNiO}_{3}$ is directly connected to a rebalancing of the distribution of charges on the $\mathrm{Ni}$ sites. We also find direct evidence that the dynamics of the $\mathrm{Ni}$ and $\mathrm{Nd}$ sublattices can be completely decoupled depending on the degree of excitation of the system. We speculate that tracking the ultrafast dynamics of rare earth magnetic moments may find broad applicability in the study of dynamically evolving internal magnetic fields in out of equilibrium complex solids.

We thank M. Altarelli for useful discussions and M. Petrich and J. Harms for technical support. Portions of this research were carried out on the SXR Instrument at the Linac Coherent Light Source (LCLS), a division of SLAC National Accelerator Laboratory and an Office of Science user facility operated by Stanford University for the US Department of Energy. The SXR Instrument is funded by a consortium whose membership includes the LCLS, Stanford University through the Stanford Institute for Materials Energy Sciences (SIMES), Lawrence Berkeley National Laboratory (LBNL), University of Hamburg through the BMBF priority program FSP 301, and the Center for Free Electron Laser Science (CFEL). We acknowledge support from the Diamond Light Source for the provision of experimental beamtime under Awards No. SI7285 and No. SI8207-1. A.D.C. acknowledges financial support by the SNSF. Y.D.C. acknowledges support by the Advanced Light Source (ALS). ALS is supported by Department of Energy under Contract No. DE-AC0205CH11231. Work at Brookhaven National Laboratory is supported by the Department of Energy under Contract No. DE-AC02-98CH10886.
*Present address: Department of Physics, University of York, United Kingdom.

${ }^{1}$ E. Beaurepaire, J.-C. Merle, A. Daunois, and J.-Y. Bigot, Phys. Rev. Lett. 76, 4250 (1996).

${ }^{2}$ C. Stamm, T. Kachel, N. Pontius, R. Mitzner, T. Quast, K. Holldack, S. Khan, C. Lupulescu, E. F. Aziz, M. Wietstruk, H. A. Durr, and W. Eberhardt, Nat. Mater. 6, 740 (2007).

${ }^{3}$ C. D. Stanciu, F. Hansteen, A. V. Kimel, A. Kirilyuk, A. Tsukamoto, A. Itoh, and T. Rasing, Phys. Rev. Lett. 99, 047601 (2007).

${ }^{4}$ B. Koopmans, G. Malinowski, F. Dalla Longa, D. Steiauf, M. Fähnle, T. Roth, M. Cinchetti, and M. Aeschlimann, Nat. Mater. 9, 259 (2010).

${ }^{5}$ A. Kirilyuk, A. V. Kimel, and T. Rasing, Rev. Mod. Phys. 82, 2731 (2010).
${ }^{6}$ N. P. Duong, T. Satoh, and M. Fiebig, Phys. Rev. Lett. 93, 117402 (2004).

${ }^{7}$ L. Rettig, R. Cortes, S. Thirupathaiah, P. Gegenwart, H. S. Jeevan, M. Wolf, J. Fink, and U. Bovensiepen, Phys. Rev. Lett. 108, 097002 (2012).

${ }^{8}$ S. Wall, D. Prabhakaran, A. T. Boothroyd, and A. Cavalleri, Phys. Rev. Lett. 103, 097402 (2009).

${ }^{9}$ J. B. Goodenough, Phys. Rev. 100, 564 (1955).

${ }^{10}$ H. Ehrke, R. I. Tobey, S. Wall, S. A. Cavill, M. Först, V. Khanna, T. Garl, N. Stojanovic, D. Prabhakaran, A. T. Boothroyd, M. Gensch, A. Mirone, P. Reutler, A. Revcolevschi, S. S. Dhesi, and A. Cavalleri, Phys. Rev. Lett. 106, 217401 (2011).

${ }^{11}$ M. Först, R. I. Tobey, S. Wall, H. Bromberger, V. Khanna, A. L. Cavalieri, Y.-D. Chuang, W. S. Lee, R. Moore, W. F. Schlotter, 
J. J. Turner, O. Krupin, M. Trigo, H. Zheng, J. F. Mitchell, S. S. Dhesi, J. P. Hill, and A. Cavalleri, Phys. Rev. B 84, 241104(R) (2011).

${ }^{12}$ S. L. Johnson et al., Phys. Rev. Lett. 108, 037203 (2012).

${ }^{13}$ W. S. Lee et al., Nat. Commun. 3, 838 (2012).

${ }^{14}$ R. I. Tobey, S. Wall, M. Först, H. Bromberger, V. Khanna, J. J. Turner, W. Schlotter, M. Trigo, O. Krupin, W. S. Lee, Y.-D. Chuang, R. Moore, A. L. Cavalieri, S. B. Wilkins, H. Zheng, J. F. Mitchell, S. S. Dhesi, A. Cavalleri, and J. P. Hill, Phys. Rev. B 86, 064425 (2012).

${ }^{15}$ M. L. Medarde, J. Phys.: Condens. Matter 9, 1679 (1997).

${ }^{16}$ G. Catalan, Phase Transitions 81, 729 (2008).

${ }^{17}$ G. Catalan, R. M. Bowman, and J. M. Gregg, Phys. Rev. B 62, 7892 (2000).

${ }^{18}$ T. Katsufuji, Y. Okimoto, T. Arima, Y. Tokura, and J. B. Torrance, Phys. Rev. B 51, 4830 (1995).

${ }^{19}$ M. K. Stewart, J. Liu, M. Kareev, J. Chakhalian, and D. N. Basov, Phys. Rev. Lett. 107, 176401 (2011).

${ }^{20}$ S. Yamamoto and T. Fujiwara, J. Phys. Soc. Jpn. 71, 1226 (2002).

${ }^{21}$ U. Staub, G. I. Meijer, F. Fauth, R. Allenspach, J. G. Bednorz, J. Karpinski, S. M. Kazakov, L. Paolasini, and F. d'Acapito, Phys. Rev. Lett. 88, 126402 (2002).

${ }^{22}$ V. Scagnoli, U. Staub, M. Janousch, A. Mulders, M. Shi, G. Meijer, S. Rosenkranz, S. Wilkins, L. Paolasini, J. Karpinski, S. Kazakov, and S. Lovesey, Phys. Rev. B 72, 155111 (2005).

${ }^{23}$ I. I. Mazin, D. I. Khomskii, R. Lengsdorf, J. A. Alonso, W. G. Marshall, R. A. Ibberson, A. Podlesnyak, M. J. Martinez-Lope, and M. M. Abd-Elmeguid, Phys. Rev. Lett. 98, 176406 (2007).

${ }^{24}$ H. Park, A. J. Millis, and C. A. Marianetti, Phys. Rev. Lett. 109, 156402 (2012).

${ }^{25}$ J. L. Garcia-Munoz, M. A. G. Aranda, J. A. Alonso, and M. J. Martinez-Lope, Phys. Rev. B 79, 134432 (2009).

${ }^{26}$ T. Mizokawa, D. I. Khomskii, and G. A. Sawatzky, Phys. Rev. B 61, 11263 (2000).
${ }^{27}$ V. Scagnoli, U. Staub, Y. Bodenthin, M. Garcia-Fernandez, A. M. Mulders, G. I. Meijer, and G. Hammerl, Phys. Rev. B 77, 115138 (2008).

${ }^{28}$ F. Bartolome, J. Bartolome, and R. S. Eccleston, J. Appl. Phys. 87, 7052 (2000).

${ }^{29}$ R. Scherwitzl, P. Zubko, I. G. Lezama, S. Ono, A. F. Morpurgo, G. Catalan, and J.-M. Triscone, Adv. Mater. 22, 5517 (2010).

${ }^{30}$ V. Scagnoli, U. Staub, A. M. Mulders, M. Janousch, G. I. Meijer, G. Hammerl, J. M. Tonnerre, and N. Stojic, Phys. Rev. B 73, 100409(R) (2006).

${ }^{31}$ W. F. Schlotter et al., Rev. Sci. Instrum. 83, 043107 (2012).

${ }^{32}$ D. Doering, Y.-D. Chuang, N. Andresen, K. Chow, D. Contarato, C. Cummings, E. Domning, J. Joseph, J. S. Pepper, B. Smith, G. Zizka, C. Ford, W. S. Lee, M. Weaver, L. Patthey, J. Weizeorick, Z. Hussain, and P. Denes, Rev. Sci. Instrum. 82, 073303 (2011).

${ }^{33}$ M. K. Stewart, J. Liu, M. Kareev, J. Chakhalian, and D. N. Basov, Phys. Rev. Lett. 107, 176401 (2011).

${ }^{34}$ Y. Bodenthin, U. Staub, C. Piamonteze, M. Garcia-Fernandez, M. J. Martinez-Lope, and J. A. Alonso, J. Phys.: Condens. Matter 23, 036002 (2011).

${ }^{35}$ S. M. Butorin, C. Sathe, A. Agui, F. Saalem, J. A. Alonso, and J. Nordgren, Solid State Commun. 135, 716 (2005).

${ }^{36}$ H. Ichikawa, S. Nozawa, T. Sato, A. Tomita, K. Ichiyanagi, M. Chollet, L. Guerin, N. Dean, A. Cavalleri, S. Adachi, T. Arima, H. Sawa, Y. Ogimoto, M. Nakamura, R. Tamaki, K. Miyano, and S. Koshihara, Nat. Mater. 10, 101 (2011).

${ }^{37}$ A. D. Caviglia, R. Scherwitzl, P. Popovich, W. Hu, H. Bromberger, R. Singla, M. Mitrano, M. C. Hoffmann, S. Kaiser, P. Zubko, S. Gariglio, J.-M. Triscone, M. Först, and A. Cavalleri, Phys. Rev. Lett. 108, 136801 (2012).

${ }^{38}$ I. Radu, K. Vahaplar, C. Stamm, T. Kachel, N. Pontius, H. A. Dürr, T. A. Ostler, J. Barker, R. F. L. Evans, R. W. Chantrell, A. Tsukamoto, A. Itoh, A. Kirilyuk, T. Rasing, and A. V. Kimel, Nature (London) 472, 205 (2011). 